\title{
openheart Cilostazol increases patency and reduces adverse outcomes in percutaneous femoropopliteal revascularisation: a meta-analysis of randomised controlled trials
}

\author{
Alexandre M Benjo, ${ }^{1}$ Daniel C Garcia, ${ }^{2}$ J Stephen Jenkins, ${ }^{1}$ \\ Rhanderson M N Cardoso, ${ }^{2}$ Taina P Molina, ${ }^{3}$ Georges E El-Hayek, ${ }^{4}$ \\ Girish N Nadkarni, ${ }^{5}$ Emad F Aziz, ${ }^{4}$ James J Dinicolantonio, ${ }^{6}$ Tyrone Collins ${ }^{1}$
}

To cite: Benjo AM, Garcia DC, Jenkins JS, et al. Cilostazol increases patency and reduces adverse outcomes in percutaneous femoropopliteal revascularisation: a metaanalysis of randomised controlled trials. Open Heart 2014;: : 000154.

doi:10.1136/openhrt-2014000154

Received 6 June 2014 Revised 11 September 2014 Accepted 1 October 2014

CrossMark

For numbered affiliations see end of article.

Correspondence to Dr Alexandre M Benjo; abenjo@ochsner.org

\section{ABSTRACT}

Background: Cilostazol is an oral antiplatelet agent currently indicated for treatment of intermittent claudication. There is evidence that cilostazol may reduce femoropopliteal restenosis after percutaneous endovascular intervention.

Methods: We searched PubMed, Scopus and Cochrane databases from 1966 through September 2013 for randomised controlled trials (RCTs) evaluating the addition of cilostazol to standard care in patients receiving femoropopliteal endovascular treatment. Restenosis, target lesion revascularisation and combined adverse outcomes (death, revascularisation and amputation) within 1-2 years postprocedure were evaluated

Results: Of 205 articles, three RCTs were included in the analysis. The pooled data provided a total of 396 patients, 195 of whom received cilostazol. When compared to standard medical therapy alone, cilostazol significantly reduced the risk of restenosis (risk difference $-0.20 ; 95 \% \mathrm{Cl}-0.29$ to $-0.11 ; \mathrm{p}<0.0001$; number needed to treat 5), target lesion revascularisation (risk difference $-0.17 ; 95 \% \mathrm{Cl}-0.25$ to $-0.09 ; p<0.0001$; number needed to treat 6 ). Death and amputation were not different in between groups.

Conclusions and limitation: Cilostazol significantly increases femoropopliteal patency and decreases adverse outcomes in percutaneous endovascular intervention. However, further RCTs are needed because of limited sample size; this meta-analysis represents the best current evidence.

\section{INTRODUCTION}

Peripheral arterial disease (PAD) is highly prevalent and has been demonstrated to increase cardiovascular mortality by 15 -fold in individuals with severe symptomatic large-vessel disease. ${ }^{1}$ Nevertheless, it still remains an underdiagnosed and undertreated condition. ${ }^{2}$ Femoropopliteal lesions account for nearly

\section{KEY MESSAGES}

What is already known about this subject?

- Cilostazol could decrease restenosis rates after endovascular therapy for peripheral artery disease, but the current randomised controlled trials evaluating this have small sample size.

What does this study add?

- We sought to improve the current level of evidence by conducting a systematic review and metaanalysis of cilostazol and outcomes after endovascular therapy for peripheral artery disease. We discovered that compared to standard medical therapy alone, cilostazol significantly reduced the risk of restenosis and target lesion revascularisation without any significant side effects.

How might this impact on clinical practice?

- Our study provides the best current evidence for using cilostazol after endovascular therapy in peripheral artery disease as well as a rationale for a large, multicentre randomised controlled trial.

three quarters of lower extremity PAD. ${ }^{3} 4$ Revascularisation is required for patients with critical limb ischaemia. The most recent guidelines on the management of femoropopliteal PAD have expanded the indications for endovascular therapy (EVT) to the detriment of surgical revascularisation. ${ }^{5-7}$

Antiplatelet therapy is recommended after EVT for PAD. ${ }^{5}$ Cilostazol is a phosphodiesterase-3 inhibitor indicated for the treatment of intermittent claudication. ${ }^{58}$ The potential benefits of cilostazol after EVT for PAD are not addressed in current guidelines. Therefore, we decided to perform a systematic literature review and meta-analysis of the outcomes of randomised controlled trials (RCTs) that compare cilostazol to 
standard therapy alone after EVT for femoropopliteal PAD disease. Cilostazol could potentially improve procedural outcomes at a low cost, as it has been demonstrated to be cost-effective for the treatment of intermittent claudication due to PAD. ${ }^{9} 10$

\section{MATERIALS AND METHODS \\ Search strategy}

We systematically searched PubMed, Scopus and Cochrane Central Register of Controlled Trials for RCTs from 1966 to September 2013, which compared cilostazol to standard medical therapy in patients with femoropopliteal EVT. The review was performed in accordance with established methods for systematic reviews in cardiovascular medicine. ${ }^{11}$ The following medical subject heading terms were included for a MEDLINE search and adapted for other databases as needed: "cilostazol" AND ("peripheral arterial disease" OR "endovascular therapy" OR "femoropopliteal"). In addition to searching databases, reference lists of all included studies, meta-analyses and reviews were manually searched, including unpublished data. There was neither language nor patient population size restriction for the search.

\section{Data extraction}

Two authors independently completed data extraction after following defined search criteria and quality assessment. They obtained data from tables, text and graphs. When the data were presented in percentage the absolute values were calculated. Disagreements were resolved by consensus after review by the senior author of the study.

\section{Selection criteria}

Inclusion criteria were RCTs with (1) a direct comparison between patients treated with cilostazol and a control group who received standard medical therapy alone; (2) patients with femoropopliteal lesions due to PAD; (3) patients who received EVT; and (4) reports of the primary and secondary outcomes. Exclusion criteria were (1) studies that only reported outcomes of cilostazol, without a direct comparison to a control group; (2) observational studies; (3) non-randomised clinical trials; and (4) overlapping patient population, identified by studies developed over the same period of time with common authors or common study centres. In this case, only the study with a greater number of patients was included.

\section{Outcomes}

The primary outcome studied was 1-2-year incidence of restenosis after endovascular treatment defined by Doppler. Secondary outcomes of interest were 1-2-year target lesion revascularisation and combined major adverse outcomes, which included death, target lesion revascularisation, surgical revascularisation and amputations. When major combined outcomes were not presented as defined it was calculated adding the individual outcomes.

\section{Statistical analysis}

Meta-analysis was performed according to recommendations of the Cochrane Collaboration and the Quality of Reporting of Meta-analyses (QUORUM) guidelines and PRISMA statement using Review Manager (RevMan) V.5.2 version (Copenhagen, Nordic Cochrane Centre,

Figure 1 Studies selection.

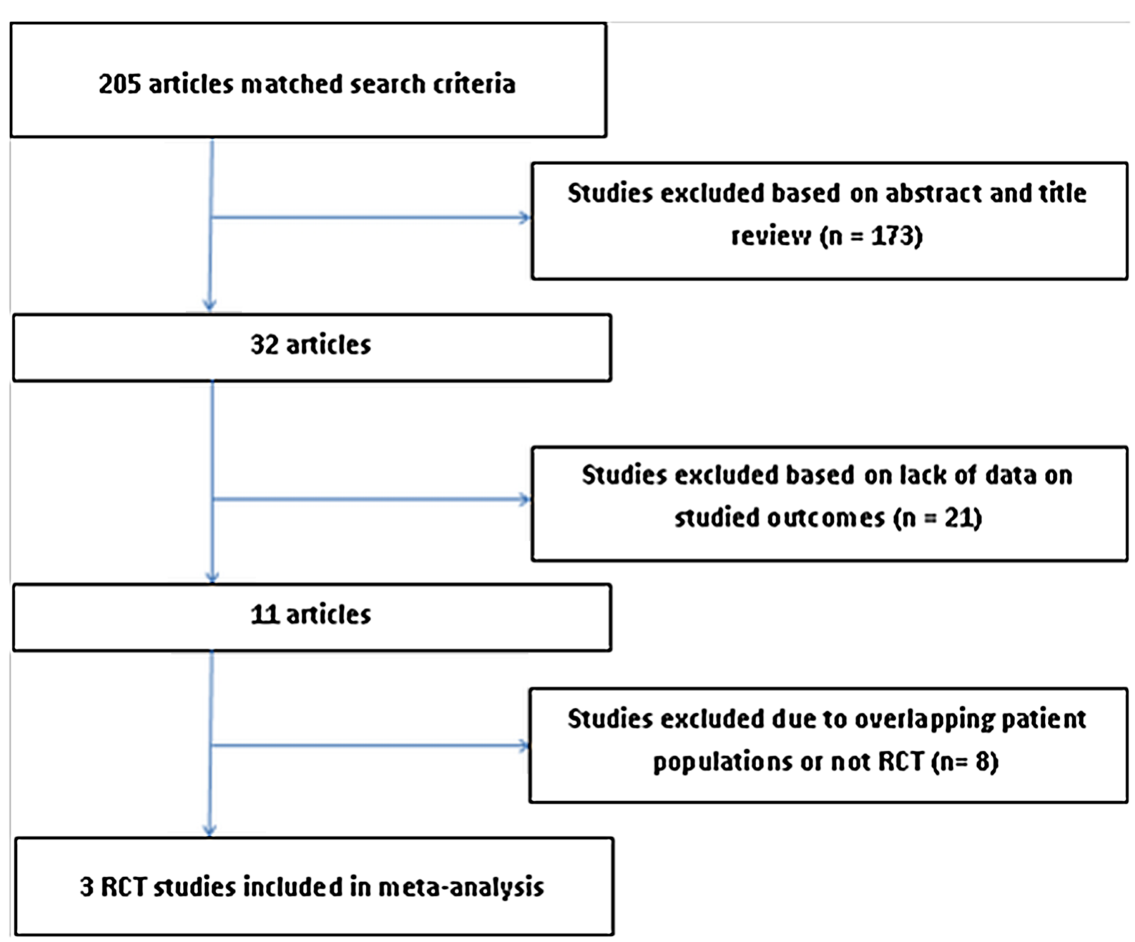


Table 1 Characteristics of studies included in the meta-analysis

\begin{tabular}{ll}
\hline Author & lida et al ${ }^{14}$ \\
Follow-up & 24 months \\
Inclusion criteria & 1. De novo FP lesions $>50 \%$ \\
& $\begin{array}{l}\text { 2. Occlusion without inflow lesions } \\
\text { 3. Outflow lesions of below-the-knee arteries } \\
>1 \text { vessel runoff }\end{array}$ \\
& 4. Symptomatic PAD with claudication (Fontain \\
& 2,3 or 4)
\end{tabular}

Control group 1. Aspirin (100 mg/day)+ticlopidine (200 mg/day)

Intervention

group

1. Aspirin (100 mg/day) and cilostazol (200 mg/ day)

Outcomes

definition

1. Lesion patency: peak systolic velocity ratio $>2.4$ by DUS

Endovascula

procedure

After balloon inflation for at least $1 \mathrm{~min}$

self-expanding stent was done if:

1. Pressure gradient $>10 \mathrm{~mm} \mathrm{Hg} \mathrm{OR}$

2. $>30 \%$ residual stenosis OR

3. flow-limiting dissection

A nitinol stent (Luminexx, CR Bard, Murray Hill,

$\mathrm{NJ}$ ) or cobalt metallic stent (Wallstent, Boston

Scientific, Natick, Mass) with the diameter $1 \mathrm{~mm}$

larger than the reference diameter was used

Stents of $6 \mathrm{~mm}$ in diameter were used in most

cases lida et $\mathrm{a} 1^{16}$

12 months

1. Patients with symptomatic PAD greater than Rutherford 1 screened by non-invasive tests to detect limb ischaemia and the presence of de novo FP lesions

1. Treated with coronary DES

2. Heart failure symptoms with systolic or diastolic dysfunction evaluated by cardiac echocardiography

3. Inflow aortoiliac lesions

4. FP lesions with severe calcification

5. Poor below-the-knee runoff defined as number of below-the-knee runoff $<1$

1. Aspirin ( $100 \mathrm{mg} /$ day)

2. Patients who received stents were also treated with a thienopyridine

1. Aspirin ( $100 \mathrm{mg} /$ day) and cilostazol $(200 \mathrm{mg} /$ day $)$

2. Patients who received stents were also treated with a thienopyridine

1. Target lesion revascularisation: reintervention performed for $>50 \%$ diameter stenosis identified by angiography within $5 \mathrm{~mm}$ of the target lesion after documentation of recurrent symptoms of PAD

2. Angiographic restenosis: recurrence of $\geq 50 \%$ diameter stenosis; a peak systolic velocity ratio of $>2.0$ on Duplex ultrasonography

After balloon inflation for at least $1 \mathrm{~min}$, stent was done if:

1. Flow-limiting dissection OR

2. Pressure gradient $>10 \mathrm{~mm} \mathrm{Hg}$ OR

3. $>30 \%$ residual stenosis

Patients received SMART stents (Cordis Corp,

Miami Lakes, Florida, USA) with a diameter $1 \mathrm{~mm}$

larger than the reference vessel diameter
Soga et al

24 months

1. Symptoms not improved by pharmacotherapy or exercise therapy

2. Age $>18$ years and $<80$ years old

3. $\mathrm{ABI}<0.9$

4. DS $>50 \%$ by visual estimate on angiography

1. Patients with previous lower extremity bypass surgery

2. Previous EVT in the femoropopliteal artery

3. Acute onset limb ischaemia

4. Rutherford category 4,5 or 6

1. Aspirin (81-100 mg/day) and ticlopidine (200 mg/day)

1. Aspirin (81-100 mg/day), ticlopidine (200 mg/day) and cilostazol (200 mg/ day)

1. Target lesion: treated segment from $10 \mathrm{~mm}$ proximal to $10 \mathrm{~mm}$ distal

2. TLR: any repeat EVT for restenosis or other complication of the target lesion with a $\%$ DS of $>50 \%$ in angiography

3. Restenosis: peak systolic velocity ratio of $\geq 2.4$ on Duplex ultrasonography

After balloon inflation for at least $1 \mathrm{~min}$, self-expanding stent was done if:

1. Angiographic residual stenosis of $>30 \%$ OR

2. Flow-limiting dissection

A commercially available self-expandable stent was used

Stent type was determined by the operators, and the stent size was chosen to be 1-

$2 \mathrm{~mm}$ larger than the vessel diameter determined

ABI, Ankle Brachial Index; DES, drug-eluting stent; DS, diameter of stenosis; EVT, endovascular therapy; DUS, distal ultrasound; FP, femoropopliteal lesion; MI, myocardial infarction; PAD, peripheral arterial disease; SMART, stent: Cordis Corp, Miami Lakes; TLR, target lesion revascularisation. 
The Cochrane Collaboration, 2012). ${ }^{12}{ }^{13}$ Pooled treatment effects were estimated using risk difference (RD) with the Mantel-Haenszel method. We calculated the number needed to treat (NNT) according to the recommendations of the Cochrane Collaboration. ${ }^{13}$ Heterogeneity was assessed using $\chi^{2}$ tests and $\mathrm{I}^{2}$ statistic; we defined $\mathrm{I}^{2}<25 \%$ as low heterogeneity according to the Cochrane Handbook of Systematic Reviews. We performed fixed effect analysis when $\mathrm{I}^{2}$ was less than $25 \%$ or $\mathrm{p}$ value at least 0.10 ; otherwise we used random effect. We assessed quality for each included trial according to the methods of the Cochrane Collaboration. ${ }^{13}$ All included studies were controlled trials and were considered high quality.

\section{RESULTS}

The process of study selection is shown in figure 1 . Initial MEDLINE search using a systematic approach yielded 205 studies. The search in EMBASE and Cochrane registries did not yield additional studies. Through a review of titles and abstracts, 173 studies were rejected due to lack of relevance to our meta-analysis. The remaining 32 articles were reviewed and assessed for satisfaction of the inclusion or exclusion criteria. Three studies met all criteria and were included in this analysis.

The first study ${ }^{14}$ had a follow-up period of 24 months and compared cilostazol/aspirin with ticlopidine/aspirin in 200 patients with femoropopliteal lesions. At 12 and 24 months, the cilostazol/aspirin group reduced restenosis rates ( $18 \%$ vs $43 \%$ and $27 \%$ vs $52 \%$, respectively). A smaller study in $2009^{15}$ also found similar results with restenosis rates $(43 \%$ vs $70.3 \%)$. These findings were validated in a larger multicentre study in 2013 with 200 patients ${ }^{16}$ that demonstrated an angiographic restenosis rate at 12 months of $20 \%$ in cilostazol group versus $49 \%$ in the non-cilostazol group.

In order to obtain pooled estimates, a total of 396 patients were included in this analysis. Following EVT for femoropopliteal PAD lesions, 195 individuals were treated with cilostazol and standard medical therapy, whereas 201 received standard medical therapy alone, which included aspirin and a thienopyridine. The main characteristics of individual studies can be found in table 1. Of note, stent restenosis by Doppler was defined as a peak systolic velocity ratio $>2.4$ in Iida $e t a l^{14}$ and Soga $e t a l^{15}$ and $>2$ in Iida et $a l^{16}$

Table 2 illustrates baseline characteristics of populations in the individual studies. All baseline variables including stenting ratio were similar in all studies with the exception of preprocedural Ankle Brachial Index (ABI) in Iida $e t a l^{16}$; despite both groups having the ABI on the moderate disease range it was higher on the cilostazol group (0.71 vs 0.66$)$.

For the primary outcome, as seen in figure 2, the follow-up revealed a significantly reduced incidence of restenosis in patients who received cilostazol in addition

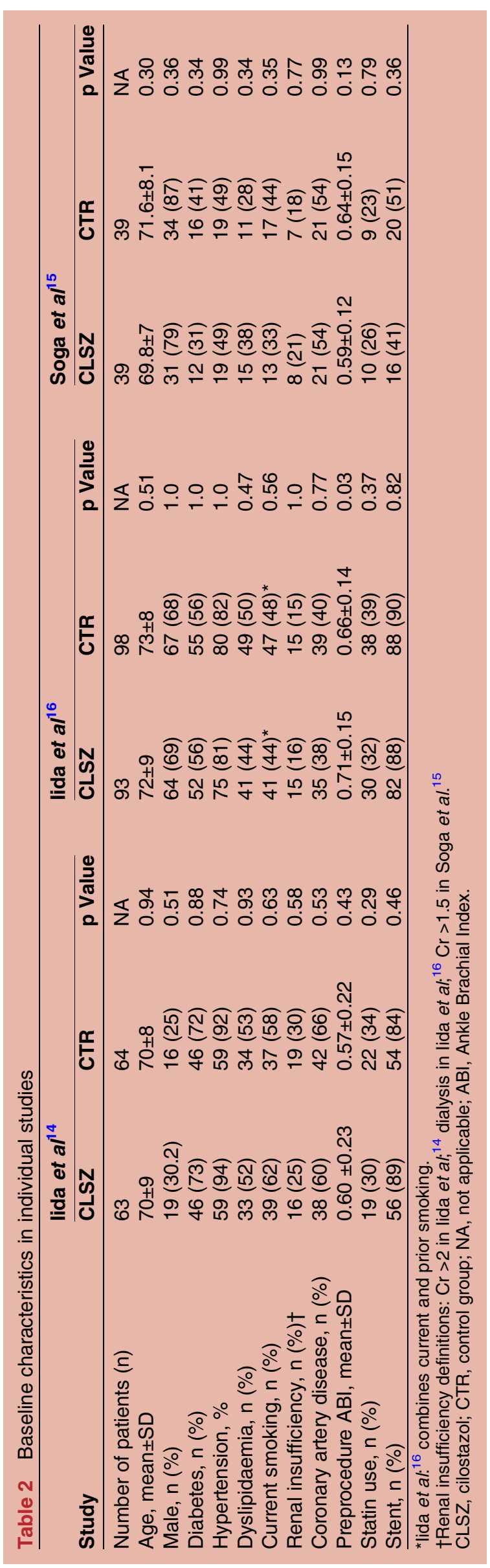




\begin{tabular}{|c|c|c|c|c|c|c|c|c|}
\hline \multirow[b]{2}{*}{ Study or Subgroup } & \multicolumn{2}{|c|}{ Cilostazol } & \multicolumn{2}{|c|}{ Control } & \multirow[b]{2}{*}{ Weight } & \multirow{2}{*}{ Risk Difference } & \multirow{2}{*}{\multicolumn{2}{|c|}{$\begin{array}{c}\text { Risk Differ ence } \\
\text { M-H, F ixell, } 95 \% \mathrm{Cl}\end{array}$}} \\
\hline & Events & Total & Events & Total & & & & \\
\hline Lida. 2008 & 12 & 63 & 24 & 64 & $32.1 \%$ & $-0.18[-0.34,-0.03]$ & $-\square$ & \\
\hline Lida.2013 & 18 & 93 & 38 & 98 & $48.2 \%$ & $-0.19[-0.32,-0.07]$ & - & \\
\hline Soga.2009 & 17 & 39 & 26 & 39 & $19.7 \%$ & $-0.23[-0.45,-0.02]$ & & \\
\hline Total $(95 \% \mathrm{Cl})$ & & 195 & & 201 & $100.0 \%$ & $-0.20[-0.29,-0.11]$ & 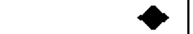 & \\
\hline Total events & 47 & & 88 & & & & & \\
\hline $\begin{array}{l}\text { Heterogeneity: } \mathrm{ChF} \\
\text { Test for overall effec }\end{array}$ & $\begin{array}{l}12, \mathrm{df}= \\
=4.38(f\end{array}$ & $\begin{array}{l}(P=0 \\
<0.0\end{array}$ & $0.94) ; 1^{2}=$ & & & & $\begin{array}{lc} & 1 \\
-1 & -0.5 \\
\text { Favors Cilostazol }\end{array}$ & $\begin{array}{|cc|}0 & 1 \\
\text { Favors Control } & 1\end{array}$ \\
\hline
\end{tabular}

Figure 2 Twelve-month to 24-month incidence of restenosis.

to standard medical (RD $-0.20 ; 95 \%$ CI -0.29 to -0.11 ; $\mathrm{p}<0.0001 ; \mathrm{NNT} 5$ ). This was reflected on a reduced need for target lesion revascularisation (RD $-0.17 ; 95 \%$ CI -0.25 to $-0.09 ; \mathrm{p}<0.0001$; NNT 6 ) with a NNT of 5 for both outcomes (figure 3). We also conducted a sensitivity analysis utilising OR as the primary pooled estimate and the effect size did not change. Death and amputations did not statistically differ between the two groups.

\section{DISCUSSION}

The potential benefit of cilostazol following EVT for femoropopliteal PAD was investigated in this study. Our meta-analysis of RCTs revealed that 75 of 201 patients not treated with cilostazol have developed restenosis within 12-24 months and that the chance of restenosis may be mitigated in more than $50 \%$ with cilostazol. This result is particularly significant when taken into account that cilostazol is a generic and inexpensive drug that has been shown to be a cost-effective drug in other clinical scenarios, such as treatment of intermittent claudication and secondary prevention of cerebral infarction ${ }^{9} 10$ and that the benefit is obtained with a small NNT.

One-year incidence of restenosis after balloon angioplasty of femoropopliteal lesions has been reported as high as $64 \% .{ }^{17}{ }^{18}$ Sirolimus-eluting stents ${ }^{19-21}$ and endovascular brachytherapy ${ }^{22}{ }^{23}$ have failed to demonstrate a long-term decrease in the restenosis incidence following lower extremity EVT for PAD, but paclitaxel-eluting stents have shown increased patency and a lower rate of events. ${ }^{23}$ Nevertheless, patency has greatly improved since the introduction of nitinol stents. ${ }^{18} 24$ When compared to percutaneous transluminal angioplasty, the use of nitinol stenting decreased the 12-month restenosis incidence from $81.3 \%$ to $36.7 \%$ in a RCT. $^{18}$ Nevertheless, the results of this meta-analysis suggest that patency rates can be further increased with cilostazol in addition to nitinol stents, given that stent use did not differ among cilostazol and control groups, as illustrated in table 2.

Cilostazol has been shown to decrease restenosis and repeat revascularisation after percutaneous coronary intervention in patients with coronary artery disease, without an increased bleeding risk. ${ }^{25}$ Similarly, in this meta-analysis, target lesion revascularisation occurred in about every third patient on the control group over a 12-24 month follow-up. The chance of requiring a repeat target lesion revascularisation was reduced in more than $60 \%$ with the use of cilostazol. The combined incidence of death, revascularisation and amputation was also significantly reduced in the cilostazol group but it was mostly driven by the reduced need for revascularisation.

The main mechanism for cilostazol-mediated decrease in restenosis and target lesion revascularisation after EVT is likely inhibition of intimal hyperplasia. Cilostazol has been shown to suppress neointimal hyperplasia in animal models. ${ }^{26}{ }^{27}$ Furthermore, RCTs have demonstrated that triple antiplatelet therapy with aspirin, clopidogrel and cilostazol was more effective than dual therapy alone in suppressing intimal hyperplasia. ${ }^{28} \mathrm{~A}$ second mechanism for improved outcomes in the cilostazol group is vasodilation. Studies have shown that cilostazol increases walking distance in patients with PAD and improves ankle-brachial index due to vasodilation. ${ }^{29-31}$ Moreover, cilostazol mediates an in-vitro inhibition of smooth muscle cell proliferation. ${ }^{32}$ Whether this effect contributes to improved EVT procedural outcomes is unknown. Cilostazol also inhibits platelet aggregation.

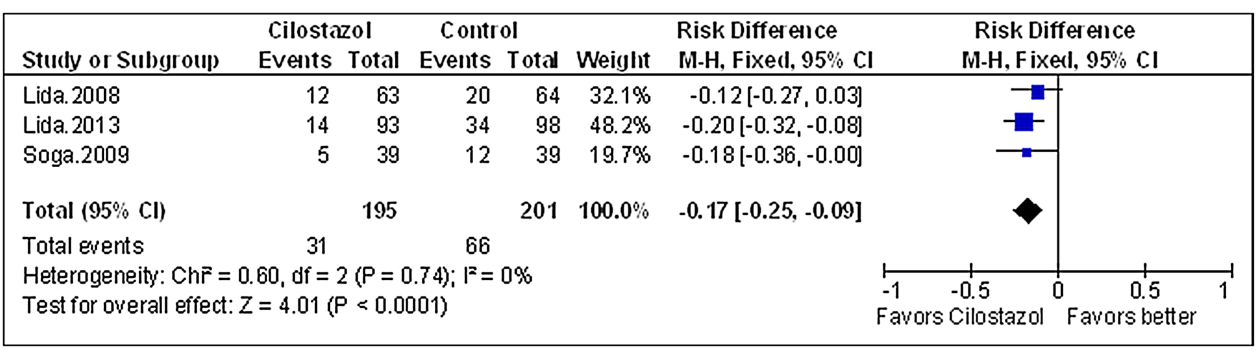

Figure 3 Twelve-month to 24-month incidence of target lesion revascularisation. 
This meta-analysis has some limitations. Owing to the limited number of studies the sample size is small. However, despite the limited sample size, our meta-analysis represents the best available evidence for the use of cilostazol to increase femoropopliteal patency after endovascular intervention. As with any metaanalysis, our study is subject to publication bias that is potentially mitigated by the exclusive use of RCTs. Although the time frame of outcomes varied from 12 to 24 months, most cases of restenosis following EVT for femoropopliteal lesions occurred from 6 to 12 months, ${ }^{33}$ a time frame that was included in this study. The available data are exclusively from Asian populations and it is possible that our results apply exclusively to Asian populations, but that is unlikely due to prior positive cilostazol studies in other populations. Finally, regarding the antiplatelet properties of cilostazol, bleeding complications could not be assessed given that this outcome was not reported in all included studies and this could be considered to be an important limitation of our analysis. Only Soga's study attempted comparing major bleeding in dual versus triple therapy but no patients had events. Although not reported in these studies, there is robust evidence that when cilostazol is used in combination with dual antiplatelet therapy for other indications, there is no increased risk of bleeding compared to dual antiplatelet therapy alone. For example, in an RCT with patients with acute coronary syndrome undergoing stenting, triple therapy had similar bleeding events compared with dual antiplatelet therapy. ${ }^{34}$

In summary, in our meta-analysis of RCTs, cilostazol following endovascular treatment for femoropopliteal PAD was shown to significantly reduce restenosis, target lesion revascularisation and combined adverse outcomes when compared to a standard therapy control group. Large RCTs are urged to confirm our findings.

\section{Author affiliations}

${ }^{1}$ Division of Interventional Cardiology, Ochsner Medical Center, New Orleans, Louisiana, USA

${ }^{2}$ Department of Internal Medicine, University of Miami, Miami, Florida, USA

${ }^{3}$ Department of Internal Medicine, Centro Universitario Luziadas School of Medicine, Santos, Brazil

${ }^{4}$ Department of Cardiology, St. Luke's-Roosevelt Hospital Center at Mount Sinai, New York, USA

${ }^{5}$ Division of Nephrology, Icahn School of Medicine at Mount Sinai, New York, USA

${ }^{6}$ Cardiology Department, St. Luke's Mid America Heart Institute, Kansas City, Missouri, USA

Contributors AMB, DCG, JSJ and TC were involved in the conception and study design. AMB, JSJ, RMNC and TPM were involved in the literature review. GEE-H, GNN, EFA and JJD were involved in the data collection. GNN, GEE-H, EFA and TPM were involved in the statistical analysis. AMB, DCG, GNN and TC were involved in the revising manuscript critically for important intellectual content. AMB, DCG, RMNC, TPM, GEE-H, GNN, EFA, JJD and TC were involved in the final approval of the manuscript submitted.

Competing interests No authors have any relationship with industry related to this manuscript.

Provenance and peer review Not commissioned; externally peer reviewed.
Data sharing statement No additional data are available.

Open Access This is an Open Access article distributed in accordance with the Creative Commons Attribution Non Commercial (CC BY-NC 4.0) license, which permits others to distribute, remix, adapt, build upon this work noncommercially, and license their derivative works on different terms, provided the original work is properly cited and the use is non-commercial. See: http:// creativecommons.org/licenses/by-nc/4.0/

\section{REFERENCES}

1. Criqui $\mathrm{MH}$, Langer $\mathrm{RD}$, Fronek $\mathrm{A}$, et al. Mortality over a period of 10 years in patients with peripheral arterial disease. $N$ Engl $J$ Med 1992;326:381-6.

2. Tattersall MC, Johnson HM, Mason PJ. Contemporary and optimal medical management of peripheral arterial disease. Surg Clin North Am 2013;93:761-78, vii.

3. Zeller T. Current state of endovascular treatment of femoro-popliteal artery disease. Vasc Med 2007;12:223-34.

4. Balzer JO, Thalhammer A, Khan V, et al. Angioplasty of the pelvic and femoral arteries in PAOD: results and review of the literature. Eur J Radiol 2010;75:48-56.

5. Norgren L, Hiatt WR, Dormandy JA, et al. Inter-Society Consensus for the Management of Peripheral Arterial Disease (TASC II). Eur J Vasc Endovasc Surg 2007;33(Suppl 1):S1-75.

6. Rooke TW, Hirsch AT, Misra S, et al. American College of Cardiology Foundation; American Heart Association Task Force; Society for Cardiovascular Angiography and Interventions; Society of Interventional Radiology; Society for Vascular Medicine; Society for Vascular Surgery. ACCF/AHA focused update of the guideline for the management of patients with peripheral artery disease (updating the 2005 guideline). Vasc Med 2011;16:452-76

7. Tendera M, Aboyans V, Bartelink ML, et al. ESC Guidelines on the diagnosis and treatment of peripheral artery diseases: document covering atherosclerotic disease of extracranial carotid and vertebral, mesenteric, renal, upper and lower extremity arteries: the task force on the diagnosis and treatment of peripheral artery diseases of the European Society of Cardiology (ESC). Eur Heart $J$ 2011;32:2851-906.

8. Thompson PD, Zimet R, Forbes WP, et al. Meta-analysis of results from eight randomized, placebo-controlled trials on the effect of cilostazol on patients with intermittent claudication. Am J Cardiol 2002;90:1314-19.

9. Squires $\mathrm{H}$, Simpson $\mathrm{E}$, Meng $\mathrm{Y}$, et al. A systematic review and economic evaluation of cilostazol, naftidrofuryl oxalate, pentoxifylline and inositol nicotinate for the treatment of intermittent claudication in people with peripheral arterial disease. Health Technol Assess 2011;15:1-210.

10. Inoue T, Kobayashi M, Uetsuka $\mathrm{Y}$, et al. Pharmacoeconomic analysis of cilostazol for the secondary prevention of cerebral infarction. Circ J 2006;70:453-8.

11. Biondi-Zoccai GG, Testa L, Agostoni P. A practical algorithm for systematic reviews in cardiovascular medicine. Ital Heart $J$ 2004;5:486-7.

12. Moher D, Liberati A, Tetzlaff J, et al; PRISMA Group. Preferred reporting items for systematic reviews and meta-analyses: the PRISMA statement. BMJ 2009;339:b2535.

13. Higgins JPT, Green S, eds. Cochrane handbook for systematic reviews of interventions version 5.1.0 (Updated March 2011). The Cochrane Collaboration, 2011.

14. lida $\mathrm{O}$, Nanto $\mathrm{S}$, Uematsu $\mathrm{M}$, et al. Cilostazol reduces restenosis after endovascular therapy in patients with femoropopliteal lesions. $J$ Vasc Surg 2008;48:144-9.

15. Soga Y, Yokoi H, Kawasaki T, et al. Efficacy of cilostazol after endovascular therapy for femoropopliteal artery disease in patients with intermittent claudication. J Am Coll Cardiol 2009;53:48-53.

16. lida $\mathrm{O}$, Yokoi $\mathrm{H}$, Soga $\mathrm{Y}$, et al. Cilostazol reduces angiographic restenosis after endovascular therapy for femoropopliteal lesions in the Sufficient Treatment of Peripheral Intervention by Cilostazol study. Circulation 2013;127:2307-15

17. Minar E, Pokrajac B, Maca T, et al. Endovascular brachytherapy for prophylaxis of restenosis after femoropopliteal angioplasty: results of a prospective randomized study. Circulation 2000;102:2694-9.

18. Laird JR, Katzen BT, Scheinert D, et al. Nitinol stent implantation versus balloon angioplasty for lesions in the superficial femoral artery and proximal popliteal artery: twelve-month results from the RESILIENT randomized trial. Circ Cardiovasc Interv 2010;3:267-76.

19. Duda SH, Pusich B, Richter G, et al. Sirolimus-eluting stents for the treatment of obstructive superficial femoral artery disease: six-month results. Circulation 2002;106:1505-9. 
20. Duda SH, Bosiers M, Lammer J, et al. Drug-eluting and bare nitinol stents for the treatment of atherosclerotic lesions in the superficial femoral artery: long-term results from the SIROCCO trial. $J$ Endovasc Ther 2006;13:701-10.

21. Duda SH, Bosiers M, Lammer J, et al. Sirolimus-eluting versus bare nitinol stent for obstructive superficial femoral artery disease: the SIROCCO II trial. J Vasc Interv Radiol 2005;16:331-8.

22. van Tongeren $\mathrm{RB}$, van Sambeek MR, van Overhagen $\mathrm{H}$, et al. Endovascular brachytherapy for the prevention of restenosis after femoropopliteal angioplasty. Results of the VARA Trial. J Cardiovasc Surg 2005;46:437-43.

23. Dake MD, Ansel GM, Jaff MR, et al. Sustained safety and effectiveness of paclitaxel-eluting stents for femoropopliteal lesions: 2-year follow-up from the Zilver PTX randomized and single-arm clinical studies. J Am Coll Cardiol 2013;61:2417-27.

24. Schillinger M, Sabeti S, Loewe $\mathrm{C}$, et al. Balloon angioplasty versus implantation of nitinol stents in the superficial femoral artery. $N$ Engl J Med 2006;354:1879-88.

25. Biondi-Zoccai GG, Lotrionte M, Anselmino M, et al. Systematic review and meta-analysis of randomized clinical trials appraising the impact of cilostazol after percutaneous coronary intervention. Am Heart J 2008;155:1081-9.

26. Park $\mathrm{SW}, \mathrm{Cha} \mathrm{IH}, \mathrm{Kim} \mathrm{CH}$, et al. Improved patency of transjugular intrahepatic portosystemic shunt: the efficacy of cilostazol for the prevention of pseudointimal hyperplasia in swine TIPS models. Cardiovasc Intervent Radiol 2007;30:719-24.

27. Kudo FA, Kondo Y, Muto A, et al. Cilostazol suppresses neointimal hyperplasia in canine vein grafts. Surg Today 2009;39:128-32.
28. Min PK, Jung JH, Ko YG, et al. Effect of cilostazol on in-stent neointimal hyperplasia after coronary artery stenting: a quantative coronary angiography and volumetric intravascular ultrasound study. Circ J 2007:71:1685-90.

29. Beebe HG, Dawson DL, Cutler BS, et al. A new pharmacological treatment for intermittent claudication: results of a randomized, multicenter trial. Arch Intern Med 1999;159:2041-50.

30. Dawson DL, Cutler BS, Meissner MH, et al. Cilostazol has beneficial effects in treatment of intermittent claudication: results from a multicenter, randomized, prospective, double-blind trial. Circulation 1998;98:678-86.

31. Money SR, Herd JA, Isaacsohn JL, et al. Effect of cilostazol on walking distances in patients with intermittent claudication caused by peripheral vascular disease. J Vasc Surg 1998;27:267-74; discussion 274-265.

32. Takahashi S, Oida K, Fujiwara R, et al. Effect of cilostazol, a cyclic AMP phosphodiesterase inhibitor, on the proliferation of rat aortic smooth muscle cells in culture. J Cardiovasc Pharmacol 1992;20:900-6.

33. Lammer J, Bosiers M, Zeller T, et al. First clinical trial of nitinol self-expanding everolimus-eluting stent implantation for peripheral arterial occlusive disease. J Vasc Surg 2011;54: 394-401.

34. Han Y, Li Y, Wang S, et al. Cilostazol in addition to aspirin and clopidogrel improves long-term outcomes after percutaneous coronary intervention in patients with acute coronary syndromes: a randomized, controlled study. Am Heart J. 2009;157:733-9. 